\title{
Implementation of the Direct $S(\alpha, \beta)$ Method in the KENO Monte Carlo Code
}

\author{
Shane W. D. Hart ${ }^{\mathrm{a}, *}$, G. Ivan Maldonado ${ }^{\mathrm{b}}$ \\ ${ }^{a}$ Oak Ridge National Laboratory \\ ${ }^{b}$ The University of Tennessee
}

\begin{abstract}
The Monte Carlo code KENO contains thermal scattering data for a wide variety of thermal moderators. These data are processed from Evaluated Nuclear Data Files (ENDF) by AMPX and stored as double differential probability distribution functions. The method examined in this paper uses $S(\alpha, \beta)$ probability distribution functions derived from the ENDF data files directly instead of being converted to double differential cross sections. This allows the size of the cross section data on the disk to be reduced substantially amount. KENO has also been updated to allow interpolation in temperature on these data so that problems can be run at any temperature. Results are shown for several simplified problems for a variety of moderators. In addition, benchmark models based on the KRITZ reactor in Sweden were run, and the results are compared with the previous versions of KENO without the Direct $S(\alpha, \beta)$ method. Results from the Direct $S(\alpha, \beta)$ method compare favorably with the original results obtained using the double differential cross sections. Sampling the data increases the run-time of the Monte Carlo calculation, but memory usage is decreased substantially.
\end{abstract}

Keywords: $\quad$ Monte Carlo, Direct $S(\alpha, \beta)$, thermal scattering, KENO

\section{Introduction}

Cross sections for use in continuous energy (CE) Monte Carlo codes are generally provided by evaluated nuclear data format (ENDF) files. These data files provide cross 5 sections for a variety of reactions and nuclear isotopes. One of the more important reactions is scattering, as this is what slows down neutrons and enables them to reach the low (thermal) energy range. Outside of the thermal range, neutrons can always be assumed to only downscatter 10 that is, always lose energy in a collision. However, in the range of a few $\mathrm{eV}$, the thermal motion of the nuclei can no longer be ignored, and neutrons can easily gain energy in a collision. For certain moderating materials such as hydrogen in water, a neutron tends to interact with an 15 aggregate of atoms rather than with single nucleus. These atoms can be in molecular or crystalline materials, and this further complicates the scattering process. (Trkov et al. 2011) ]

\footnotetext{
* Corresponding author

Email addresses: hartsw@ornl.gov (Shane W. D. Hart), imaldona@utk.edu (G. Ivan Maldonado)

This manuscript has been authored by UT-Battelle, LLC under Contract No. DE-AC05-00OR22725 with the U.S. Department of Energy. The United States Government retains and the publisher, by accepting the article for publication, acknowledges that the United States Government retains a non-exclusive, paid-up, irrevocable, world-wide license to publish or reproduce the published form of this manuscript, or allow others to do so, for United States Government purposes. The Department of Energy will provide public access to these results of federally sponsored research in accordance with the DOE Public Access Plan (http://energy.gov/downloads/doe-publicaccess-plan).
}

For inelastic scattering in the thermal range, ENDF files provides $S(\alpha, \beta)$ data. Inelastic scattering is generally represented by the thermal scattering law and is defined for a moderating molecule or crystal by

$$
\begin{aligned}
\frac{d^{2} \sigma}{d E^{\prime} d \Omega}(E \rightarrow & \left.E^{\prime}, \mu, T\right)= \\
& \frac{\sigma_{b}}{4 \pi k T}\left(\frac{E}{E^{\prime}}\right)^{1 / 2} \exp \left(-\frac{\beta}{2}\right) S(\alpha, \beta, T),
\end{aligned}
$$

where $\sigma_{b}$ is the scattering cross section for a neutron incident on a bound nucleus, $k T$ is the temperature in $\mathrm{eV}$, and $S(\alpha, \beta, T)$ is a scattering function that depends on the detailed dynamics and structure of the scattering material.

To use the $S(\alpha, \beta)$ data, two dimensionless quantities must be defined. The dimensionless momentum transfer, $\alpha$, is defined by

$$
\alpha=\frac{E+E^{\prime}-2 \mu \sqrt{E^{\prime} E}}{A k T},
$$

$\mathrm{E}=$ incident neutron energy $(\mathrm{eV})$,

$\mathrm{E}^{\prime}=$ scattered neutron energy $(\mathrm{eV})$,

where, $\mu=$ cosine of the scattering angle,

$\mathrm{A}=$ atomic mass,

$\mathrm{kT}=$ temperature $(\mathrm{eV})$ and

The dimensionless energy transfer, $\beta$, is defined by

$$
\beta=\frac{E-E^{\prime}}{k T},
$$

where positive values represent downscattering events. 
Currently for the Monte Carlo code KENO Goluoglu et al. 2011) in SCALE (Bowman, 2011), the $S(\alpha, \beta)$ values are used to calculate the double differential cross section in Eq. (1). These cross sections can then be used to create cumulative distribution functions (CDFs) for sampling in 65 KENO. This paper discusses a method that allows for the fhe $S(\alpha, \beta)$ data directly instead of first calculating the double differential cross sections. By using the data directly, the storage space required for the CE libraries can be significantly reduced at the expense of a slightly longer 70 run time. In order to be used by KENO, the $S(\alpha, \beta)$ CDFs

35 need to be calculated and stored. This is accomplished by the creation and use of a new program called $\mathrm{S} \alpha \beta \mathrm{RE}$ which is added to AMPX (Dunn and Greene, 2002).

ENDF files provide the thermal moderating data at a fixed number of temperatures. In order to to obtain tem40 peratures at intermediate values, an interpolation scheme implemented using a unit-based interpolation scheme similiar to that described in (Hart et al. 2014). The $S(\alpha, \beta)$ data are only used for inelastic scattering. Elastic scattering in the thermal region must be modeled using the 45 original double differential scattering data.

\section{Direct $S(\alpha, \beta)$ Method}

Scattering law data are stored in ENDF files as a set of function values, $S$, over a grid of discrete $\alpha$ and $\beta$ values. By storing the scattering data in this way, the storage 50 requirement is minimized. Unfortunately, the data generally must be processed and put into another form useful for computational methods before it can be used. As an example, the AMPX module Y12 uses the $S(\alpha, \beta)$ values to calculate the double differential cross section, $\sigma\left(\mu, E \rightarrow E^{\prime}\right)$.

55 Bischoff first suggested the direct $S(\alpha, \beta)$ method by exploiting the compact format of the $S(\alpha, \beta)$ data through generating probability distribution functions (PDFs) in both $\alpha$ and $\beta$ to drastically lower the storage cost Bischoff $^{75}$ et al. 1972).

Using the definitions for $\alpha$ and $\beta$, the double differential cross section is defined as

$$
\sigma\left(E \rightarrow E^{\prime}, \mu\right)=\frac{\sigma_{b}}{2 k T} \sqrt{\frac{E^{\prime}}{E}} e^{\beta / 2} S(\alpha, \beta)
$$

where $\sigma_{b}$ is the bound cross section unique to each element.

(Ballinger, 1995) noticed that a transformation matrix could be obtained given the definitions of $\alpha$ and $\beta$ in terms of energy and angle, where

$$
\left[\begin{array}{ll}
\frac{d \alpha}{d E} & \frac{d \alpha}{d \mu} \\
\frac{d \beta}{d E} & \frac{d \beta}{d \mu}
\end{array}\right]=\left[\begin{array}{cc}
\frac{1-\mu \sqrt{\frac{E^{\prime}}{E}}}{A k T} & \frac{-2 \sqrt{E^{\prime} E}}{A k T} \\
\frac{1}{k T} & 0
\end{array}\right] .
$$

Rewriting the double differential cross section in terms of $\alpha$ and $\beta$ using the determinant of the transformation matrix ${ }^{80}$ yields

$$
\sigma(\alpha, \beta)=\frac{\sigma_{b} A k T}{4 E} S(\alpha, \beta) e^{\beta / 2}
$$

By noticing that the leading term is a constant, this double differential cross section can be integrated over all $\alpha$ and $\beta$ values to obtain a scattering cross section as a function of energy.

There is no efficient way to directly sample $\alpha$ and $\beta$ from Eq. (6). Therefore, as described in Section 3. CDFs and PDFs are generally constructed and then sampled to determine the scattered neutron energy by first sampling a $\beta$ followed by sampling an $\alpha$. Integration over both $\alpha$ and $\beta$ is required to convert the scattering law data into a set of CDFs. By doing this, the CDF for $\alpha$ can be decoupled from the incident energy which will reduce the storage requirement for the data.

The two associated CDFs are

$$
P\left(\beta_{i} \mid E, T\right)=\int_{0}^{\beta_{i}} C e^{\beta / 2} \int_{\alpha_{\min }}^{\alpha_{\max }} S(\alpha, \beta, T) \mathrm{d} \alpha \mathrm{d} \beta,
$$

where $C$ is a constant and thus does not affect the calculation of the CDF, and

$$
P\left(\alpha_{i} \mid \beta, E, T\right)=\int_{0}^{\alpha_{i}} \frac{S\left(\alpha^{\prime}, \beta, T\right)}{\int_{\alpha_{\text {min }}}^{\alpha^{\max }} S(\alpha, \beta, T) \mathrm{d} \alpha} \mathrm{d} \alpha^{\prime}
$$

As mentioned previously, the $\alpha$ CDF can be decoupled from the incident energy by storing $\mathrm{F}$ factors defined as

$$
F\left(\alpha_{i}, \beta_{j}\right)=\int_{0}^{\alpha_{i}} S\left(\alpha^{\prime}, \beta_{j}\right) e^{\beta_{j} / 2} d \alpha^{\prime}
$$

and calculating the $\alpha$ CDFs on the fly from

$$
P\left(\alpha \mid \beta_{j}\right)=\frac{F\left(\alpha, \beta_{j}\right)-F\left(\alpha_{\min }, \beta_{j}\right)}{F\left(\alpha_{\max }, \beta_{j}\right)-F\left(\alpha_{\min }, \beta_{j}\right)} .
$$

Constructing the $\alpha$ CDFs on the fly is the key for making the direct $S(\alpha, \beta)$ method memory conservative.

In order to calculate $\alpha_{\min }$ and $\alpha_{\max }$, one uses the knowledge that the minimum momentum transfer occurs during a glancing collision (or $\mu=1$ ). Therefore using Eqs. (2) and (3) $\alpha_{\min }$ is

$$
\alpha_{\text {min }}=\frac{2 E-\beta k T-2 \sqrt{E^{2}-E \beta k T}}{A k T}
$$

and $\alpha_{\max }(\mu=-1)$ is

$$
\alpha_{\max }=\frac{2 E-\beta k T+2 \sqrt{E^{2}-E \beta k T}}{A k T} .
$$

Once the $\alpha$ and $\beta$ CDFs are constructed, it is straightforward to sample a $\beta$ from the distribution and use it to calculate an $\alpha$ value. First a $\beta$ is sampled from the $\beta$ distribution and used, along with the incident energy, to sample an $\alpha$ from the constructed $\alpha$ distribution described in Eq. (10). The sampled $\alpha$ and $\beta$ values are then converted into scattering cosine and exit energy using the definitions given in Eqs. (2) and (3). 


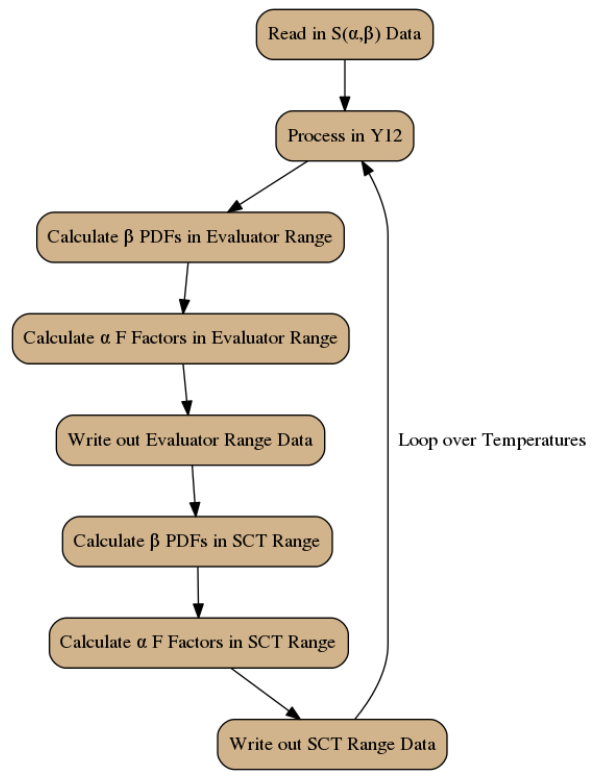

Figure 1: Program flow of SABRE.

$\beta$ CDFs are stored on an incident energy mesh that ${ }_{125}$ is calculated by $\mathrm{Y} 12$, and the $\alpha \mathrm{F}$ factors are stored on the resulting $\beta$ mesh. Since the $\alpha \mathrm{F}$ factors do not also need to be stored on an incident energy mesh, the memory taken up by the CDFs can be greatly reduced. However, since $\alpha$ CDFs then need to be calculated during neutron transport, the time taken to sample a neutron scattering ${ }_{130}$ event increases when using the direct $S(\alpha, \beta)$ method.

\section{Implementation}

\subsection{SABRE Module in $A M P X$}

135

In order to create the $\alpha$ and $\beta$ CDFs, a new AMPX module was created. This module implemented the methods described in Section 2 to create a binary file that is read in by KENO. This module is heavily based on the existing AMPX module Y12, which reads in the ENDF data ${ }^{140}$ file and prepares double differential cross sections. This new module, $S(\alpha, \beta)$ Reformat Engine, can be pulled into Y12 and no longer exist as a separate program.

The program flow of SABRE is shown in Fig. 1, which depicts two distinct areas of SABRE. First, the data for ${ }^{145}$ the evaluator range are processed, and then the data in the short collision time (SCT) approximation region are calculated and processed. SABRE calls Y12 to generate the incident and final energy grids to be used by the $\beta$ CDFs. The $\alpha$ grid used is that created by the evaluator ${ }^{150}$ on the ENDF file.

Generally, when evaluations are performed and placed into ENDF files, the evaluator assigns the maximum energy for which the $S(\alpha, \beta)$ values should be used. This maximum energy typically is anywhere from 2 to $4 \mathrm{eV}^{.15}$ Above that range, the SCT approximation is used. The
SCT approximation (Borgonovi, 1970) is an equation performed to directly obtain a $S(\alpha, \beta)$ value using

$$
S^{\mathrm{SCT}}(\alpha, \beta, T)=\frac{\exp \left[-\frac{(\alpha-|\beta|)^{2} T}{4 \alpha T_{\text {eff }}(T)}-\frac{|\beta|}{2}\right]}{\sqrt{4 \pi \alpha \frac{T_{\text {eff }}(T)}{T}}},
$$

10 where $T_{\text {eff }}$ is the effective temperature obtained from the ENDF file. Once the $S(\alpha, \beta)$ values are obtained, the calculation of the CDFs proceeds as before.

Currently the $\beta$ grid created depends on the final energy grid obtained from Y12. That is, for a specific incident energy, a $\beta$ point is created for every calculated exit energy. This leads to a final exit energy grid with the same density as that produced by Y12 and currently used in KENO for the double differential cross section scattering. The grid is fairly dense, and therefore computationally inefficient for sampling. Therefore, there is potential for thinning the grid by removing points that can be recalculated through linear interpolation (within some error tolerance). The $\alpha$ grid is the same as that created by the evaluator in the ENDF file. No work has been done to expand or thin this grid. The total size of the $S(\alpha, \beta)$ binary data file is generally about one-quarter the size of a similar double differential CDF data file.

\section{2. $K E N O$}

Implementing the direct $S(\alpha, \beta)$ method into KENO is relatively straightforward. When a collision occurs, KENO determines the type of reaction that will occur by using the one-dimensional cross sections. Currently, if the reaction will be an incoherent inelastic scatter, KENO will sample the double differential CDFs by sampling a cosine followed by an exit energy. The properties of the neutron are adjusted, and the history continues. With the direct $S(\alpha, \beta)$ method, subroutine sample_alpha was created to sample $\alpha$, and subroutine sample_beta was created to sample $\beta$. For temperature interpolation, the existing Doppler broadening routines were expanded to include a subroutine to interpolate on the $S(\alpha, \beta) \mathrm{CDF}$, and a new module was created to aid in the interpolation.

The original data specify that one should interpolate on the double differential cross sections and not $S(\alpha, \beta)$. Our results (shown in Section 4 show that there is minimal difference between broadening the double-differential cross section or $S(\alpha, \beta)$, indicating that for the investigated moderators the tempereature mesh for $S(\alpha, \beta)$ is dense enough.

Sampling of $\beta$ was performed first so that the calculated $\beta$ could be used to determine $\alpha_{\min }$ and $\alpha_{\max }$ for the $\alpha$ on the fly sampling. Sampling the $\beta$ is performed using a similar method as that used to sample the exit energy for the double differential cross section method. If the incoming energy matches an energy on the energy grid exactly, then a random number is sampled, and the $\beta$ is calculated from the CDFs for that energy. If the incoming energy is between two energy points on the incident energy grid, 
then one random number is sampled and used to generate $\beta$ values using the bounding incident energies. The final beta is then determined by linear interpolation on the incoming energy.

After $\beta$ is determined, the exit energy can be determined by using Eq. (3) in the form

$$
E^{\prime}=E-\beta k T
$$

Determining $\alpha$ (and therefore $\mu$, the scattering cosine) ${ }^{210}$ is slightly more involved than calculating $\beta$. The steps to sample $\alpha$ are:

1. sample a random number,

2 . interpolate between the $\beta$ values to obtain an $\mathrm{F}$ factor grid for all $\alpha$ values,

3. calculate $\alpha_{\min }$ and $\alpha_{\max }$ using Eqs. (11) and (12),

4. calculate the CDFs using Eq. (10) up to and including the random number, and

5. determine $\alpha$ from the random number and CDFs.

Once $\alpha$ is determined, $\mu$ can be determined by using Eq. (2) in the form

$$
\mu=\frac{E+E^{\prime}-\alpha A k T}{2 \sqrt{E E^{\prime}}} .
$$

A new subroutine was created that controls the logic for prebroadening the $S(\alpha, \beta)$ CDFs. First, the $\alpha \mathrm{F}$ factors is no need for unit base interpolation due to the fact that the CDFs are calculated as needed when given $\beta$. Next, the $\beta$ values are interpolated by looping over all incoming energies, creating $\beta$ CDFs at that energy if they do not exist, and interpolating to the desired temperature.

It is necessary to use a unit-base interpolation scheme when interpolating $\beta$ values on temperature. After a new $\beta$ CDF panel is created, it can be used to overwrite the existing data so that the data are now temperature corrected.

\section{Results}

\subsection{Testing}

As a test to verify the accuracy of the direct $S(\alpha, \beta)$ method, a variety of scatters off of various moderators were simulated, and the results were tallied into exit energy and cosine bins. For each test, several million scattering events were tallied. After each scatter, the neutron was reset to the initial energy so that a distribution of exit energies and cosines could be obtained.

Fig. 2 shows the results of scattering 10 million neutrons off of hydrogen in water, using both the double differential scattering cross sections CDFs and the direct $S(\alpha, \beta)$ method. As can be seen in Fig. 2a, the exit energy distribution from the direct $S(\alpha, \beta)$ method almost overlays the ${ }^{220}$ distribution obtained using the current double differential cross section approach. There is a bit more structure to the direct $S(\alpha, \beta)$ results, as evidenced by the dips and rises around $0.8 \mathrm{eV}$ and $0.9 \mathrm{eV}$. For the scattering angle results in Fig. 2b, the double differential results are hampered by a 32 equiprobable bin limit chosen at library creation. The limit was originally chosen to make the on-disk library small while still preserving sufficient fidelity. One can clearly see 32 distinct steps in which the probability is the same. Due to the on-the-fly method used for sampling the $\alpha$ value, the scattering angle distribution is a smooth curve.

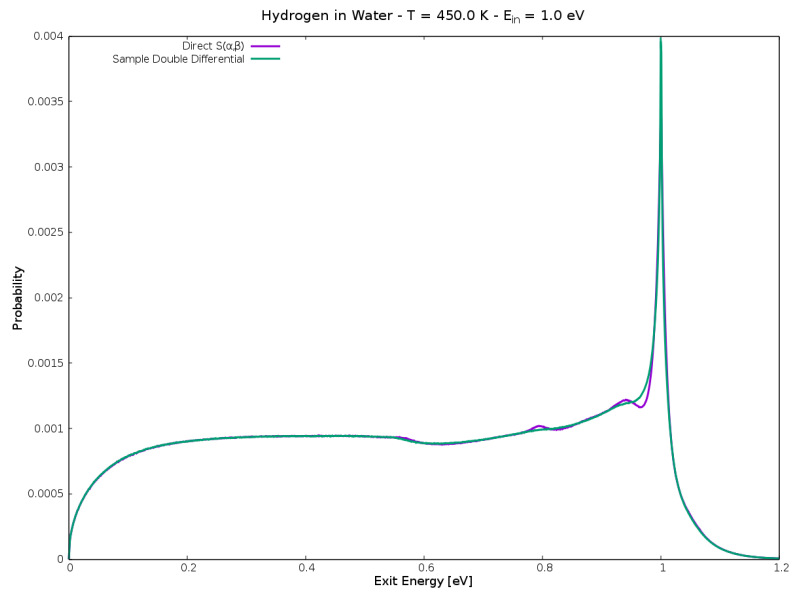

(a) Exit energy

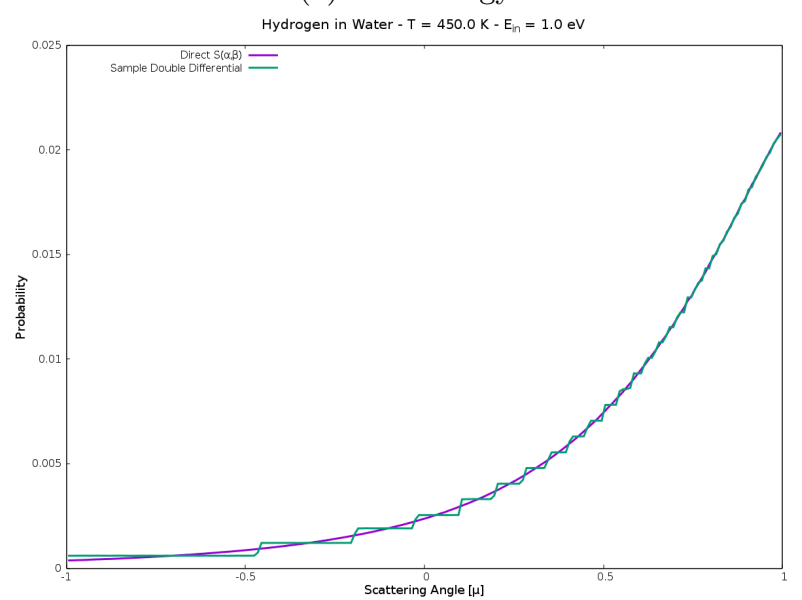

(b) $\mu$

Figure 2: Neutron scattering off of hydrogen in water at $E_{\text {in }}=1 \mathrm{eV}$, $T=450 \mathrm{~K}$.

The main drawback of using the direct $S(\alpha, \beta)$ method is that, due to constructing the $\alpha$ CDFs during the collision, each collision takes two to three times longer to sample. While this is not noticeable for a single collision, running these scattering tests with millions of neutrons leads to the run time increasing from tens of seconds to a minute or more.

More examples of scattering off of hydrogen in water are shown in Figs. 3 and 4 . Fig. 4 is particularly interesting due to the fact that $S(\alpha, \beta)$ values here are calculated using the SCT approximation in which most struc- 
ture in the PDFs disappear. This leads to a relatively flat probability distribution instead of the structure seen in the evaluation range. This is particularly noticeable when the incoming energy is near the exit energy. In the evaluation range, there is a clear peak in which $E=E^{\prime}$, whereas in the SCT range this peak is gone. There is, however, still a small peak when using the double differential cross sections. This peak is not physical and is believed to come from corrections made by AMPX to account for long tails in the CDF distribution. Regardless, as shown in the eigenvalue results, this peak has little effect on the eigenvalue.

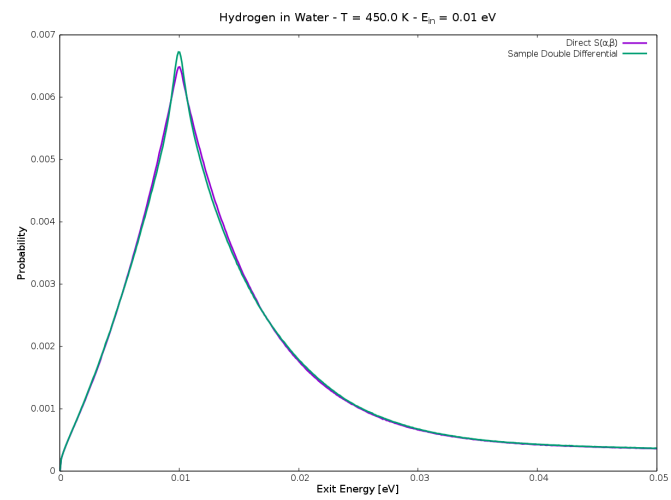

(a) Exit energy

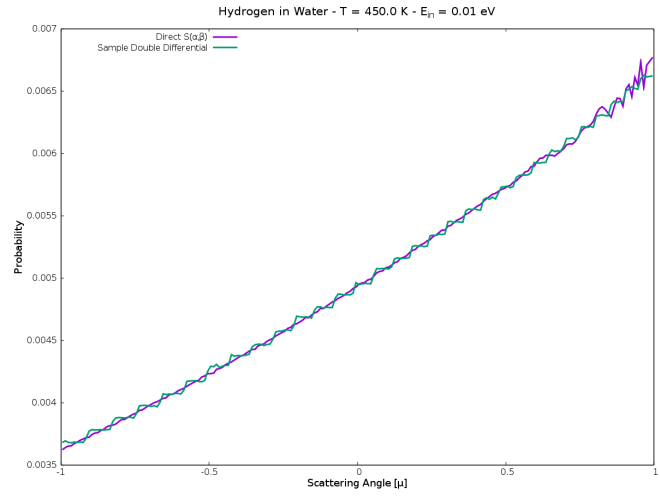

(b) $\mu$

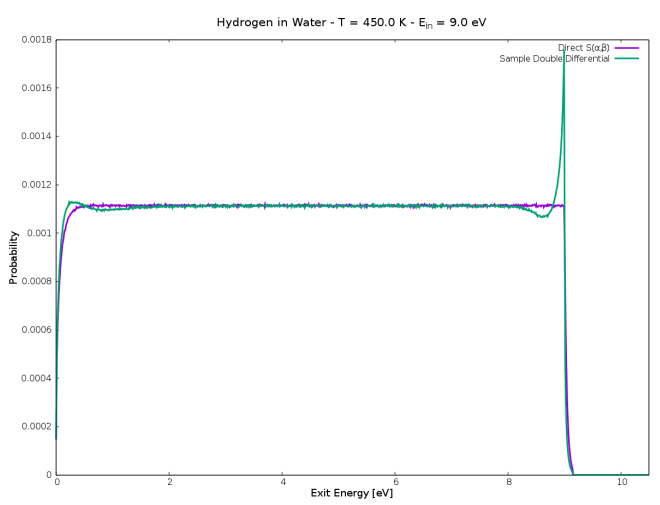

(a) Exit energy

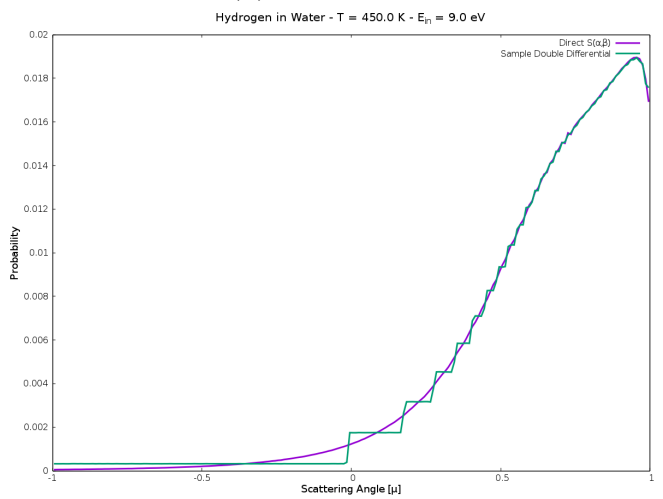

(b) $\mu$

Figure 4: Neutron scattering off of hydrogen in water at $E_{\text {in }}=9 \mathrm{eV}$, $T=450 \mathrm{~K}$.

shows the results when intermediate temperatures are added and interpolation is performed on the $S(\alpha, \beta)$ data. While the data are somewhat noisy due to the uncertainty caused by the Monte Carlo simulation, the results when using the direct $S(\alpha, \beta)$ method are generally within one or two standard deviations from using the double differential cross sections.

Figure 3: Neutron scattering off of hydrogen in water at $E_{\text {in }}=0.01$ $\mathrm{eV}, \mathrm{T}=450 \mathrm{~K}$.

Another example of a prominent thermal scattering material is graphite. Results showing graphite scatter\$260 are in Figs. 5 and 6 . Again, the structure of the $S(\alpha, \beta)$ values can be see inside the evaluation range (which is up to $2 \mathrm{eV}$ for graphite). Above $2 \mathrm{eV} \mathrm{SCT}$ is used.

\subsection{Pincell}

A simple pin cell model was created using $3.1 \%$ enriched $\mathrm{UO}_{2}$ with a Zircalloy clad. Fig. 7a shows the results obtained using the direct $S(\alpha, \beta)$ method at the library temperatures (no interpolation). One-dimensional

Doppler broadening (KENO option $d b x=1$ ) must be ${ }^{270}$ used because the moderator contains ${ }^{16} \mathrm{O}$, which must be Doppler broadened to the correct temperature. Fig. $7 \mathrm{~b}$

\subsection{Timing and Data Library Sizes}

Due to the heterogeneous nature of the computing cluster at the University of Tennessee Department of Nuclear Engineering (http://necluster.engr.utk.edu/), it can be difficult to obtain timing results for many cases because they may be run on computers of vastly differing speeds. Table 1 shows timing results for a few cases that were run on the same or similar nodes. As can be seen from the table, there is a definite slow-down when direct $S(\alpha, \beta)$ is enabled. This can be attributed to the need to calculate the $\alpha$ CDFs during scatter sampling. The slow-down depends entirely on how many scattering events occur in the moderator. For these problems, the ratio of fuel-to-moderator is not that low, so the slow-down experienced is not that high (less than 10\%).

The main benefit of the direct $S(\alpha, \beta)$ method is that the data libraries can be smaller than those associated with the double differential method. Table 2 shows the average 


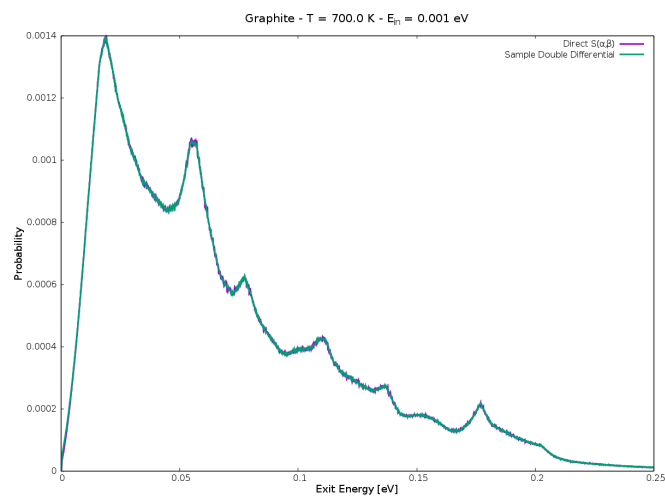

(a) Exit energy

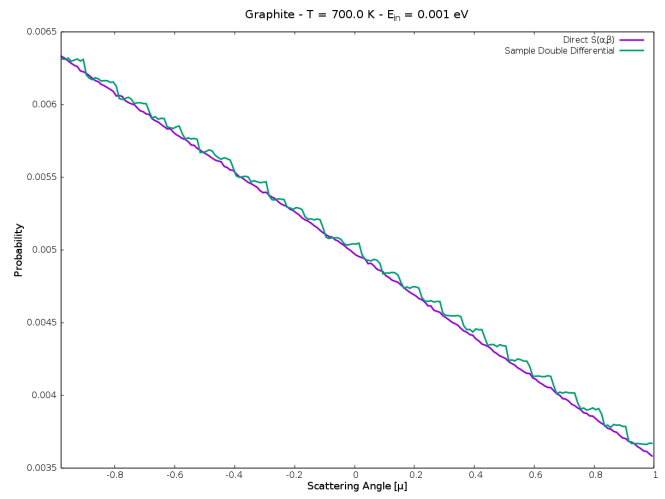

(b) $\mu$

Figure 5: Neutron scattering off of graphite at $E_{\text {in }}=0.001 \mathrm{eV}, T=$ $700 \mathrm{~K}$.

Table 1: Timing Results for Direct $S(\alpha, \beta)$ Cases

\begin{tabular}{cccc}
\hline Moderator & $\begin{array}{c}\text { Double } \\
\text { Differential } \\
{[\text { min }]}\end{array}$ & $\begin{array}{c}\text { Direct } \\
S(\alpha, \beta) \\
{[\text { min }]}\end{array}$ & $\begin{array}{c}\text { Slow-Down } \\
{[\%]}\end{array}$ \\
\hline $\mathrm{H}_{2} \mathrm{O}$ & 578 & 627 & 8.5 \\
Graphite & 741 & 807 & 8.9 \\
\hline
\end{tabular}

library size per temperature for some of the common moderators discussed in this section. It is important to keep in mind that the direct $S(\alpha, \beta)$ libraries only contain the $\alpha$ $\mathrm{F}$ factors and $\beta$ CDFs. They still depend on the some features of the original libraries, such as the one-dimensional cross sections and energy grid. The current direct $S(\alpha, \beta)$ libraries have also not been optimized. Work is needed to further thin the resultant data and remove duplicate values for the SCT range. Some testing has been performed in which libraries under one megabyte in size have been constructed.

\section{Benchmarks}

The International Handbook of Evaluated Reactor Physics Benchmark Experiments (IHERPhBE) (Committee et al.

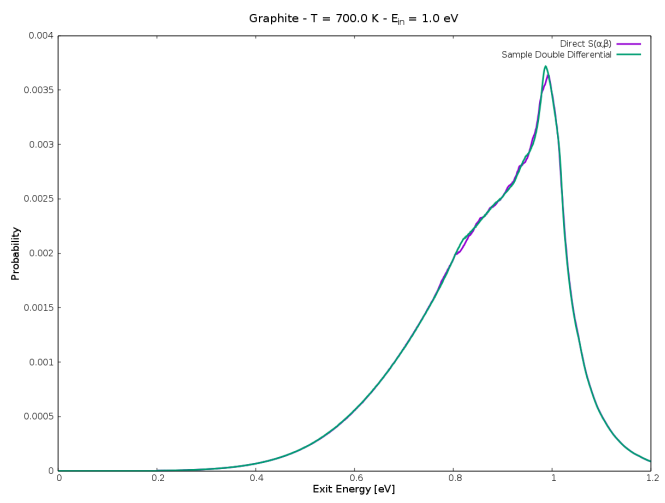

(a) Exit energy

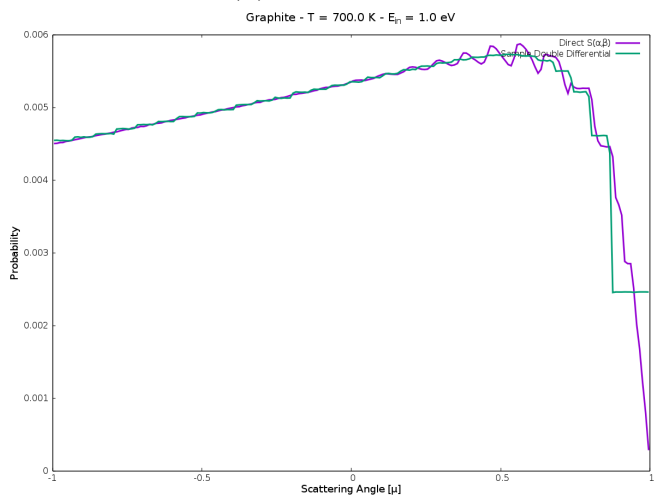

(b) $\mu$

Figure 6: Neutron scattering off of graphite at $E_{\text {in }}=1 \mathrm{eV}, T=700$ K.

2006 was prepared by a working group of experienced reactor physics personnel. It contains reactor physics benchmark specifications derived from experiments performed at 290 various nuclear experimental facilities around the world. The benchmark specifications are intended for use to validate calculation techniques. The most recent edition of the handbook contains data from 53 different experimental series performed at 31 different reactor facilities.

To test the accuracy of the temperature intperolation, benchmarks were chosen to be consistent with those in previous Doppler broadening work. (Hart et al. 2016)

Table 2: Size of Library for Direct $S(\alpha, \beta)$

\begin{tabular}{cccc}
\hline Moderator & $\begin{array}{c}\text { Double } \\
\text { Differential } \\
{[\mathrm{MB}]}\end{array}$ & $\begin{array}{c}\text { Direct } \\
S(\alpha, \beta) \\
{[\mathrm{MB}]}\end{array}$ & $\begin{array}{c}\% \text { of Double } \\
\text { Differential }\end{array}$ \\
\hline Hydrogen in Water & 21 & 5 & 24 \\
Graphite & 30 & 4 & 13 \\
Beryllium & 30 & 3 & 10 \\
Polyethylene & 17 & 3 & 18 \\
\hline
\end{tabular}




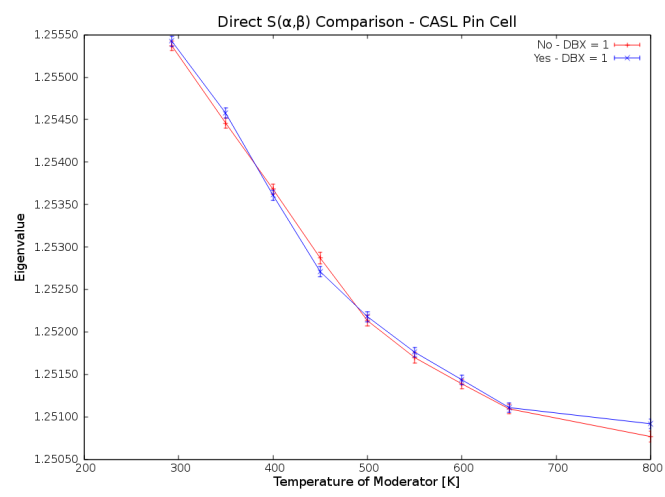

(a) $\mathrm{DBX}=1$

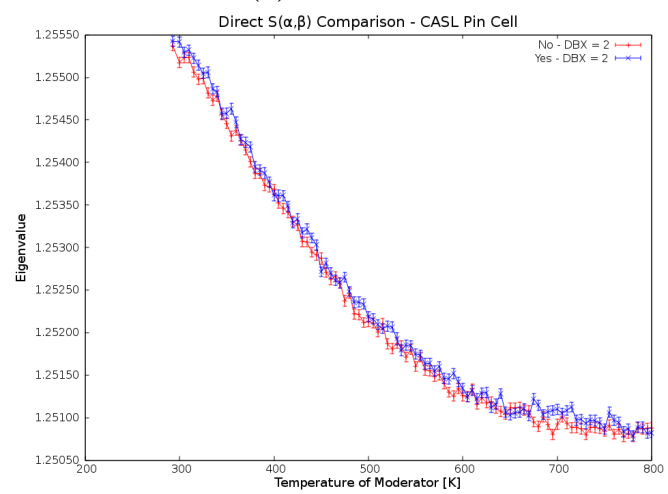

(b) $\mathrm{DBX}=2$

Figure 7: Direct $S(\alpha, \beta)$ results for pin cell (water).

\subsection{KRITZ-2:19}

The first evaluation, KRITZ-2:19, was an experiment with mixed-oxide rods at an $18.00 \mathrm{~mm}$ pitch. Criticality was obtained by controlling the boron content in the water and adjusting the water level. The square inner tank was filled with water up to the level needed to achieve criticality. The space between the inner tank and the cylindrical tank served as a dump area and was effectively filled with steam.

The experimental, measured, and benchmark-model ${ }^{360}$ $\mathrm{k}_{\text {eff }}$ are given in Table 3 . As a critical reactor, it is expected that the experimental and measured $k_{\text {eff }}$ will be The uncertainty in the measured $k_{\text {eff }}$ arises from the error in measuring the multiplication factor (used to calculate $\mathrm{k}_{\mathrm{eff}}$ ) of the reactor. The uncertainty in the ex- ${ }^{365}$ perimental $\mathrm{k}_{\mathrm{eff}}$ arises from the uncertainties of the various parameters in the experiment such as materials, temperapere, and geometry. Due to simplifications made in the model, it was determined that the benchmark $\mathrm{k}_{\mathrm{eff}}$ will actually be slightly above unity. The benchmark $\mathrm{k}_{\mathrm{eff}}$ is ${ }^{370}$ the value expected to be calculated with the Monte Carlo codes. Tables 4 and 5 show the results obtained for the old case and hot case respectively for both MCNP and KENO with and without direct $S(\alpha, \beta)$ enabled. In order to be consistent with the evaluation results, $C / E$ values $^{375}$ were calculated with the benchmark $\mathrm{k}_{\mathrm{eff}}$.

For the cold case the KENO results are consistent to obtained with MCNP. With the direct $S(\alpha, \beta)$ method enabled, the eigenvalue dips slightly, but it is still within the desired range. For the hot case, MCNP only uses temperature corrected JEFF 3.1 data, and KENO has Doppler broadening turned on. Again, the results are consistent $S(\alpha, \beta)$ method is slightly lower than the original method.

\subsection{KRITZ-2:1}

The second KRITZ evaluation, KRITZ-2:1, was a rectangular array of low enriched uranium Zircalloy-2 fuel rods in light water (Snoj and Gehin, 2009). Criticality was achieved by regulating the concentration of boron in water and by adjusting the water level.

The experimental, measured, and benchmark-model $\mathrm{k}_{\mathrm{effs}}$ are given in Table 6. The statements regarding the $\mathrm{k}_{\text {eff }}$ values made in regard to KRITZ-2:19 (Section 5.1) also apply for this KRITZ case. Tables 7 and 8 show the benchmark results from MCNP (obtained from the evaluation report) and from KENO for the cold and hot cases, respectively. MCNP used a weighted mixture of cross sections from the two closest libraries to obtain cross sections that are accurate at the desired temperatures. For the cold case, KENO was actually slightly closer to the benchmark values than MCNP. For the hot case, KENO was slightly further away from the benchmark than MCNP. Without Doppler broadening, the KENO results are particularly off the benchmark. Enabling Doppler broadening increased the $\mathrm{k}_{\text {eff }}$ by $\sim 150 \mathrm{pcm}$ and made the results closer to the benchmark. As in previous cases, enabling direct $S(\alpha, \beta)$ lowered the $\mathrm{k}_{\mathrm{eff}}$ by $\sim 10 \mathrm{pcm}$, which was generally within one standard deviation of the original KENO result.

\section{Conclusions}

Constructing and sampling cosine and exit energy distribution functions provides and effective, yet memory intensive method, of modeling thermal scattering in Monte Carlo transport codes. Alternatively, using the direct $S(\alpha, \beta)$ method would decrease memory usage at the cost of computational time. Having the ability to select which function is more important to the user at any given time would be useful and provide the user with additional ways to tweak the calculation to fit a specific machine or purpose.

The direct $S(\alpha, \beta)$ method is not new, but it has not yet been implemented in any SCALE code sequence. Adding the direct $S(\alpha, \beta)$ method to KENO involved also adding a module in AMPX, S $\alpha \beta \mathrm{RE}$, to allow for the creation of the necessary data. S $\alpha \beta \mathrm{RE}$ is currently an experimental module of the AMPX suite of tools.

Results from using the direct $S(\alpha, \beta)$ method compare favorably to those obtained using the traditional double differential sampling methods. Comparisons between the methods were obtained from simulating scattering off of hydrogen in water and carbon in graphite. Results for exit energies were close between the methods. Results for 
Table 3: Experimental, measured, and benchmark-model $\mathrm{k}_{\mathrm{eff}}$ for KRITZ-2:19.

\begin{tabular}{ccc}
\hline & Cold & Hot \\
\hline Measured & $1.0000 \pm 0.0001$ & $1.0000 \pm 0.0001$ \\
Experimental & $1.0000 \pm 0.0015$ & $1.0000 \pm 0.0019$ \\
Benchmark & $1.0077 \pm 0.0030$ & $1.0055 \pm 0.0027$ \\
\hline
\end{tabular}

Table 4: Benchmark results for KRITZ-2:19 cold case.

\begin{tabular}{ccc}
\hline & $\mathrm{k}_{\text {eff }}$ & $\frac{C}{E}-1(\%)$ \\
\hline MCNP & $1.0023 \pm 0.0001$ & -0.54 \\
JEFF 3.1 & \\
MCNP & $1.0031 \pm 0.0001$ & -0.44 \\
ENDF/B-VII.0 & \\
KENO & & \\
ENDF/B-VII.0 & $1.0027 \pm 0.0001$ & -0.50 \\
No Direct $S(\alpha, \beta)$ & \\
KENO & & \\
ENDF/B-VII.0 & $1.0024 \pm 0.0001$ & -0.52 \\
Direct $S(\alpha, \beta)$ & & \\
& & \\
\hline
\end{tabular}

Table 5: Benchmark results for KRITZ-2:19 hot case.

\begin{tabular}{ccc}
\hline & $\mathrm{k}_{\mathrm{eff}}$ & $\frac{C}{E}-1(\%)$ \\
\hline MCNP & $1.0011 \pm 0.0001$ & -0.44 \\
JEFF 3.1 & \\
KENO & \\
ENDF/B-VII.0 & $1.0010 \pm 0.0001$ & -0.45 \\
No Direct $S(\alpha, \beta)$ & \\
KENO & \\
ENDF $/$ B-VII.0 & $1.0007 \pm 0.0001$ & -0.48 \\
Direct $S(\alpha, \beta)$ & & \\
\hline
\end{tabular}

Table 6: Experimental, measured, and benchmark-model $\mathrm{k}_{\mathrm{eff}}$ for KRITZ-2:1

\begin{tabular}{ccc}
\hline & Cold & Hot \\
\hline Measured & $1.0000 \pm 0.0001$ & $1.0000 \pm 0.0001$ \\
Experimental & $1.0000 \pm 0.0018$ & $1.0000 \pm 0.0026$ \\
Benchmark & $1.0025 \pm 0.0020$ & $1.0024 \pm 0.0028$ \\
\hline
\end{tabular}

Table 7: Benchmark results for KRITZ-2:1 cold case.

\begin{tabular}{ccc}
\hline & $\mathrm{k}_{\text {eff }}$ & $\frac{C}{E}-1(\%)$ \\
\hline MCNP & $0.9975 \pm 0.0001$ & -0.50 \\
JEFF 3.1 & \\
MCNP & $0.9981 \pm 0.0001$ & -0.43 \\
ENDF/B-VII.0 & \\
KENO & & \\
ENDF/B-VII.0 & $0.9990 \pm 0.0001$ & -0.35 \\
No Direct $S(\alpha, \beta)$ & \\
KENO & & \\
ENDF $/$ B-VII.o & $0.9989 \pm 0.0001$ & -0.36 \\
Direct $S(\alpha, \beta)$ & & \\
& & \\
\hline
\end{tabular}

Table 8: Benchmark results for KRITZ-2:1 hot case.

\begin{tabular}{ccc}
\hline & $\mathrm{k}_{\mathrm{eff}}$ & $\frac{C}{E}-1(\%)$ \\
\hline MCNP & $0.9992 \pm 0.0001$ & -0.33 \\
JEFF 3.1 & \\
KENO & \\
ENDF/B-VII.0 & $0.9973 \pm 0.0001$ & -0.51 \\
No Direct $S(\alpha, \beta)$ & \\
KENO & \\
ENDF/B-VII.0 & $0.9971 \pm 0.0001$ & -0.53 \\
Direct $S(\alpha, \beta)$ & & \\
\hline
\end{tabular}


the scattering cosine were smoother when using the direct $S(\alpha, \beta)$ method due to the fact that cosines could be calculated on the fly and are not limited by the number of bins chosen at library creation.

Currently the data libaries shipped with SCALE cannot be easily extended to support the addition of new data such as that required by the $S(\alpha, \beta)$ sampling. Future work will involve modernizing the SCALE data format so that data can be easily added. In addition, there is still much work to do to optimize the direct $S(\alpha, \beta)$ CDFs. Further improvements in both speed and on-disk memory consumption could be obtained through further optimiza390 tion.

\section{Acknowledgements}

The work documented in this paper was performed with support from the U.S. Department of Energy Nuclear Criticality Safety Program.

\section{References}

Ballinger, C.T., 1995. The direct $\mathrm{S}(\alpha, \beta)$ method for thermal neutron scattering, in: Proceedings of the International. Conference on Mathematics and Computation, Reactor Physics, and Environmental Analysis, pp. 134-143.

Bischoff, F., Yeater, M., Moore, W., 1972. Monte carlo evaluation of multiple scattering and resolution effects in double-differential neutron scattering cross-section measurements. Nucl. Sci. Eng

1] 48, 266-280. URL: http://www.ans.org/pubs/journals/nse/a 22485

405 Borgonovi, G.M., 1970. Neutron Scattering Kernels Calculations at Epithermal Energies. Technical Report GA-9950. Gulf General Atomic.

Bowman, S.M., 2011. SCALE 6: Comprehensive Nuclear Safety Analysis Code System. Nucl. Technol. 174, 126.

410 Committee, N.N.S., et al., 2006. International Handbook of Evaluated Reactor Physics Benchmark Experiments. Technical Report. NEA/NSC/DOC.

Dunn, M., Greene, N., 2002. AMPX-2000: A cross-section processing system for generating nuclear data for criticality safety applications. Trans. Am. Nucl. Soc 86, 118-119.

Goluoglu, S., Petrie, L.M., Dunn, M.E., Hollenbach, D.F., Rearden, B.T., 2011. Monte carlo criticality methods and analysis capabilities in scale. Nuclear Technology 174, 214-235. Accepted for publication on August 24, 2010.

420 Hart, S., Maldonado, G.I., Celik, C., Leal, L.C., 2014. Problemdependent doppler broadening of continuous-energy cross section in the KENO Monte Carlo code, in: PHYSOR 2014, American Nuclear Society, Kyoto, Japan.

Hart, S.W.D., Celik, C., Maldonado, G.I., Leal, L., 2016. Creation of problem-dependent Doppler-broadened cross sections in the KENO Monte Carlo code. Annals of Nuclear Energy 88, 4956.

Snoj, L., Gehin, J.C., 2009. KRITZ-2:1 Experiment on Regular $\mathrm{H}_{2} \mathrm{O} /$ Fuel Pin Lattices with Low Enriched Uranium Fuel at Temperatures $248.5{ }^{\circ} \mathrm{C}$. Technical Report KRITZ-LWR-RESR-002. Jozef Sefan Institute and Oak Ridge National Laboratory.

Trkov, A., Herman, M., Brown, D., 2011. ENDF-6 Formats Manual. Technical Report. Report BNL-90365-2009 Rev. 2, Brookhaven National Laboratory, Upton, New York. 